Canadian University Music Review

Canadian University Music Review

Revue de musique des universités canadiennes

\title{
Le rythme harmonique dans la musique d'orgue de la période tonale
}

\section{Benjamen Waterhouse}

Volume 11, numéro 1, 1991

URI : https://id.erudit.org/iderudit/1014828ar

DOI : https://doi.org/10.7202/1014828ar

Aller au sommaire du numéro

\section{Éditeur(s)}

Canadian University Music Society / Société de musique des universités canadiennes

\section{ISSN}

0710-0353 (imprimé)

2291-2436 (numérique)

Découvrir la revue

Citer cet article

Waterhouse, B. (1991). Le rythme harmonique dans la musique d'orgue de la période tonale. Canadian University Music Review / Revue de musique des universités canadiennes, 11(1), 1-33. https://doi.org/10.7202/1014828ar

All Rights Reserved @ Canadian University Music Society / Société de musique des universités canadiennes, 1991
Ce document est protégé par la loi sur le droit d'auteur. L'utilisation des services d'Érudit (y compris la reproduction) est assujettie à sa politique d'utilisation que vous pouvez consulter en ligne.

https://apropos.erudit.org/fr/usagers/politique-dutilisation/ 


\title{
LE RYTHME HARMONIQUE DANS LA MUSIQUE D'ORGUE DE LA PÉRIODE TONALE
}

\author{
Benjamen Waterhouse
}

\section{Introduction}

L'importance du rythme harmonique dans la musique d'orgue

La musique occidentale se distingue des autres cultures musicales par l'importance accordée à l'harmonie, c'est-à-dire la relation « verticale » entre les notes entendues et la relation subséquente entre les " accords » ainsi formés. Notre oreille est habituée à chercher une couleur harmonique non seulement lorsque deux ou plusieurs notes sont entendues simultanément, mais aussi lorsque ces notes sont entendues successivement à la façon d'un arpège (qu'on pense, par exemple, aux Suites pourviolon seul de Bach). C'est précisément cette multiplicité d'accords potentiels qui rend extrêmement difficile le sujet que nous nous proposons de traiter ici : le rythme harmonique. Ce dernier terme est devenu d'usage courant dans la littérature musicale du $\mathrm{XX}^{\mathrm{e}}$ siècle ; l'Américain Walter Piston est peut-être le principal responsable de sa vulgarisatin et ce sont surtout des études américaines qui jettent les bases d'une définition classique que nous empruntons ici à Sessions, cité par Marks (1969: 3) : « [le rythme harmonique] est le dessin rythmique formé par les accords et les changements d'accord $\gg .{ }^{1}$

Il est évident que le point crucial de cette définition est la notion d'accord : qu'estce qui constitue un accord? À quel moment situer un changement d'accord? Si l'on admet que toute combinaison verticale de sons entendus simultanément ou successivement au cours d'une œuvre représente un accord, la moindre pièce de musique occidentale contient un nombre quasi incalculable d'accords potentiels. C'est pour cette raison qu'on retrouve dans toute étude du rythme harmonique l'idée de l'accord essentiel par opposition à l'accord décoratif, de passage, ou d'importance secondaire. La définition de Sessions continue ainsi : "Un 'changement d'harmonie' signifie nécessairement un changement de basse fondamentale " (Marks 1969:4). ${ }^{2}$ Même si cette qualification est endossée dans la plupart des méthodes d'analyse harmonique, la façon de l'appliquer et de manipuler les autres critères peut faire varier les résultats d'une analyse harmonique selon les besoins de l'analyste.

La situation particulière de notre instrument, l'orgue, a été la motivation première pour entreprendre cette étude où l'analyse n'est pas un but en soi mais

1 « [Harmonic rhythm is] the rhythmic pattern formed by the chords and chord changes ».

2 " By 'changes of harmony' is, of course, meant change of root." 
plutôt un outil concret offert à l'interprète. Des tous les instruments, l'orgue est celui pourrait le mieux soutenir l'appellation d'instrument harmonique : premièrement, à cause de sa capacité à faire entendre des sons et des accords indéfiniment ; deuxièmement, à cause des lieux extrêmement réverbérants, en général des églises, où il trouve sa place naturelle, et où le détail mélodique et les rythmes de surface s'auréolent d'échos multiples et tendent à disparaître au profit du discours harmonique. Certains mélanges de jeu, en particulier le plein-jeu ou 'plenum' de l'orgue, masquent également le côté mélodique en privilégiant le côté harmonique.

Notre problématique est donc la suivante : en acceptant la définition du rythme harmonique comme un dessin rythmique formé dans le temps par les accords essentiels d'une œuvre musicale, peut-on élaborer un système d'analyse permettant de distinguer les 'accords essentiels' des autres accords, à partir des seuls éléments fournis par la pièce elle-même ? Peut-on tenir compte, dans cette analyse écrite, des effets d'une acoustique réverbérante sur la diffusion sonore de l'orgue ? Y a-t-il un lien entre les critères de l'analyse et la façon dont l'auditeur averti distingue un déroulement harmonique à travers les multiples combinaisons harmoniques potentielles qui l'assaillent de toutes parts? Pour essayer de répondre à ces questions, nous avons choisi de présenter ici l'analyse détaillée d'une seule œuvre (le Prélude en mi mineur, BWV 548, de Bach), accompagnée d'une discussion des divers problèmes entourant l'élaboration de la grille d'analyse.

\section{Le rythme harmonique}

\section{L'analyse}

L'analyse musicale prend le plus souvent comme point de départ la partition écrite, représentation graphique d'une réalité ou d'un potentiel sonore. Les deux axes de la représentation sont, de gauche à droite, le déroulement du temps et, de bas en haut, l'échelle des hauteurs utilisées. L'utilité de la partition en analyse est de traduire un phénomène sonore temporel (la musique) en une forme intemporelle où l'on peut embrasser d'un seul coup d'œil la totalité d'une pièce ou, au contraire, se pencher sur un détail presque imperceptible à l'audition. L'unidirectionalité du temps se trouve aussi suspendue et l'analyste peut sauter impunément du 'passé' au 'futur' musical, faire des rapprochements dans le temps, voire postuler des phénomènes rétroactifs. Une technique courante en analyse est la réduction temporelle; ainsi, une pièce de quarante mesures est-elle représentée graphiquement en trois mesures, ou bien par une formule telle que $\mathrm{ABA}$; cette réduction temporelle s'accompagne d'une sélection hiérarchique des valeurs qui figureront dans la nouvelle représentation aux dépens des autres. De telles analyses sont extrêmement utiles pour l'étude de la forme en musique et pour découvrir des structures et des symétries cachées ; mais, par le fait même de l'abstraction du passage réel du temps, leur intérêt comme outil d'interprétation est limité. 
Le but de la présente étude est d'examiner la nature et la fonctionnement d'un élément de l'écriture musicale formé par l'interaction de l'harmonie avec le rythme. Toute manipulation et, surtout, toute réduction sélective du temps musical se trouvent donc exclues. De plus, comme nous démontrerons plus loin que le rythme harmonique n'est qu'un des nombreux fils qui composent le tissu rythmique, nos désirons pouvoir l'étudier sans pour autant masquer les autres fils avec lesquels il doit réagir. C'est pour ces raisons que nous avons choisi, pour présenter nos analyses, un système appelé " analyse-réseau » par Eugene Narmour (1977), système développé par Robert O. Gjerdingen (1988).

\section{L'analyse-réseau}

La caractéristique de l'analyse-réseau est de présenter une expansion, et non pas une compression, de l'information musicale de la partition. La graphie originale et, conséquemment, le déroulement temporel normal sont conservés ; vient s'ajouter un nombre variable de portées supplémentaires sur lesquelles seront isolés pour examen différents éléments de l'écriture musicale.

\section{Le tissu rythmique}

Le rythme dans la musique tonale est un domaine très vaste qui fait l'objet, depuis plusieurs années, d'investigations variées de la part des musicologues. Cependant, la complexité du sujet empêche pour l'instant la formulation d'une explication globale ; même les termes les plus élémentaires (rythme, mètre, accent, accentuation, phrase, cadence) donnent lieu à des définitions diverses, selon l'auteur consulté et son système l'analyse. Devant cet état de choses, nous avons préféré prendre comme référence l'ouvrage d'un seul auteur (Lester 1986) plutôt que de chercher à composer des définitions d'origines différentes. Il convient de souligner que l'auteur en question, loin d'imposer de façon unilatérale des définitions abstraites, s'efforce de prendre en considération et d'adopter, parfois en les modifiant, les recherches les plus récentes dans le domaine.

Le rythme d'une pièce tonale peut être comparé à un tissu complexe formé de fils multiples. Chacun de ces fils représente le rythme d'un seul paramètre. Ainsi, dans une pièce contrapuntique à trois voix, le rythme de chaque voix représente un des fils du tissu, le rythme des accords de trois sons un deuxième, le rythme des attaques simultanées dans les trois voix un troisième, le rythme des arrivées sur les points cadentiels un quatrième plus large, et ainsi de suite. Le nombre des rythmes que l'on peut découvrir est évidemment infini, selon le type d'analyse qu'on applique à la pièce ; et c'est l'interaction de ces rythmes variés, les uns agissant de concert avec le mètre, les autres s'y opposant totalement, qui crée la vie rythmique de l'œuvre.

\section{Rythme et accentuation}

Ce sont les multiples accents créés par une ligne musicale qui nous permettent de percevoir son rythme. D'après la définition de Lester, l'accent musical, contrairement à l'accent poétique qui possède une durée, est un instant qui se 
démarque du temps musical quil'entoure : il est créé par le début d'unévénement. Ainsi, au niveau le plus élémentaire, le début d'une note se démarque du silence ou de la partie soutenue d'une autre note qui le précède. C'est pour cette raison que lorsque l'on parle du rythme d'une monodie, par exemple, on parle du rythme dessiné dans le temps par l'attaque des notes. Dans un contexte plus chargé, où l'oreille doit suivre plusieurs voix, ce sont certains points saillants de chacune qui la démarqueront du contexte général. Ces 'points saillants' sont les accents créés par divers facteurs, comme :

- le début d'une cellule motivique ;

- une valeur longue qui suit des valeurs plus courtes ;

- la note la plus aiguë ou la plus grave d'un motif ou d'une phrase ; et ainsi de suite.

Le propos principal de Lester est de démontrer comment les différentes séries d'accents créés dans une ligne musicale, souvent sur la partie faible du temps telle que définie par le mètre, enrichissent le tissu rythmique de la pièce.

D'autres facteurs sont susceptibles de créer des points accentués dans l'ensemble des voix, ensemble considéré comme une sorte de 'super-voix'. En règle générale, les accents de cette 'super-voix' coïncident avec le mètre, formant ainsi un cadre rythmique duquel se démarquent les accents des voix individuelles; le résultat en est donc ce tissu rythmique dont les fils superposés créent la dynamique de l'œuvre.

Nous avons repris la classification des facteurs créateurs d'accent donnée par Lester, mais en sélectionnant ceux qui agissent, non pas sur une ligne mélodique, mais sur l'ensemble des voix, car c'est le niveau de fonctionnement du rythme harmonique dont nous suivons la trace ici.

Facteurs créateurs d'accent dans l'ensemble des voix

Comme nous l'avons noté plus haut, le début d'un événement nouveau se démarque de ce qui le précède, créant par conséquent un accent. Ainsi, le début des événements suivants, impliquant plusieurs ou toutes les voix d'une texture, marque un point d'accentuation :

- une nouvelle harmonie ;

- une harmonie plus longue suivant des changements d'harmonie rapides ;

- un changement de texture (par addition ou soustraction de voix) ;

- des attaques simultanées dans plusieurs voix.

Ces éléments créent des points d'accentuation au niveau de la texture générale de la pièce, de même que d'autres éléments mentionnés plus haut donnent une vie rythmique à une seule ligne de la texture. Tous agiront soit en concertation, soit en désaccord avec le mètre, sur lequel nous portons maintenant notre attention.

Le mètre

Le terme 'mètre' désigne l'organisation d'une suite de pulsations égales en une 
hiérarchie régulière, où une pulsation accentuée (temps fort) est suivie d'une ou de plusieurs pulsations non accentuées (temps faibles) ; les temps forts d'un niveau donné deviennent les pulsations du niveau suivant, à leur tour organisées en temps forts et temps faibles. Le résultat en est la hiérarchie rythmique classique, où les doubles croches sont groupées en deux par les croches, les croches groupées en deux par les noires, les noires par les blanches, et les blanches par les rondes. Ce sont les premières notes d'une pièce qui font entendre soit une pulsation régulière, soit une pulsation irrégulière où les valeurs sont en proportion simple de deux ou de trois, ce qui permet à l'auditeur de reconstituer la hiérarchie rythmique. Une fois la pulsation établie, certains facteurs l'organisent en mètre en indiquant les temps forts. D'après Lester, les changements harmoniques sont le prinicipal moyen utilisé pour effectuer cette organisation ; c'est seulement en l'absence d'un schéma harmonique clair que d'autres facteurs, notamment des accents de texture, entreront en jeu.

\section{L'établissement du mètre}

Lester, donc, cite l'harmonie comme l'élément le plus susceptible d'organiser la pulsation en mètre ; à défaut d'une organisation harmonique claire, ce sont d'autres facteurs, comme des attaques simultanées ou des changements de texture (nombre de voix, tessiture, etc.) qui indiquent le mètre. Le plus souvent, cependant, tous ces éléments concourront à établir de façon claire le mètre au début d'une pièce. Car c'est au départ d'une pièce que le compositeur fixe dans un climat stable le mètre, la tonalité, et les motifs thématiques de l'œuvre.

Du point de vue du mètre, il y a deux façons possibles de commencer une pièce : soit sur le premier temps d'une mesure, soit sur un autre temps, ce qui constitue un départ en anacrouse. Ce sont les facteurs d'accentuation qui situeront le temps fort : dans le premier cas, parce que ce qui suit le premier temps est moins accentué, dans le deuxième cas, parce que les premières notes sont suivies d'autres plus accentuées. Dans le Prélude en mi mineur de Bach, le premier accord à cinq voix est suivi d'un passage mélodique à une voix, donc d'une force moindre. Ce premier accord signale un temps fort ; un autre événement de même force signalera un autre temps fort et un mètre régulier sera ainsi mis sur pied. Des accords semblables au premier marquent effectivement le premier temps des mesures suivantes.

Il convient de souligner ici la relation étroite unissant le mètre d'une pièce au schéma des changements d'accord. En effet, si, au début de l'œuvre, c'est le schéma harmonique qui organise le mètre en groupant les pulsations en temps forts et en temps faibles, une fois le mètre établi, il acquiert un fonctionnement autonome et c'est lui qui aidera à définir l'importance relative d'un accord selon sa place dans la hiérarchie métrique.

\section{Ambiguïté métrique}

La compréhension du mètre par l'auditeur demeure sujette à plusieurs condi- 
tions. D'abord, comme l'ensemble de la musique tonale, le mètre est un langage codifié dont l'auditeur doit connaître les signes : on suppose donc, chez celui qui écoute, une accoutumance aux techniques de la musique tonale. Deuxièmement, la multiplicité des signes laisse dans certaines œuvres une marge d'interprétation qui peut amener l'auditeur à mal placer le mètre. Troisièmement, le compositeur peut intentionnellement introduire des éléments rythmiques qui, au départ d'une pièce, dessinent un mètre différent du mètre véritable, ou qui, au cours d'une pièce, semblent contredire le mètre établi.

Comme nous l'avons indiqué plus haut, le mètre, une fois instauré au début d'une pièce, devient une sorte de mécanique autonome susceptible de résister à des contradictions apparentes du tissu rythmique, un nouveau mètre nécessitant la même mise en place que le mètre original. Une pièce où tous les éléments concorderaient avec le mètre deviendrait vite monotone ; l'opposition d'un mètre régulier à des dessins musicaux en contretemps est un des principaux moyens de caractérisation et d'expression dont dispose le compositeur. Parfois, cette opposition devient si apparente que le mètre semble disparaître ; il ne faut pas pour autant croire à un changement de mètre, mais plutôt à une ambiguïté voulue par le compositeur. Certaines ambiguités fréquemment employées sont devenues des éléments à part entière du langage rythmique : l'hémiole, par exemple.

\section{Relation harmonique}

Notre analyse du Prélude en mi mineur de Bach vise avant tout une représentation graphique de divers éléments extraits de la trame musicale, et une représentation également graphique de ce que nous considérons être le rythme harmonique de l'œuvre.L'analyse des enchaînements harmoniques par les moyens classiques, c'est-à-dire par l'usage de chiffres arabes ou romains, nous a paru superflue. Il est cependant un aspect de l'enchaînement harmonique qui a un lien direct avec le placement métrique des accords, et c'est pour démontrer cet aspect que nous proposons le système de chiffrage décrit dans les lignes suivantes.

Nous avons adopté la technique de la basse fondamentale et lui avons réservé une portée dans notre grille d'analyse, afin de pouvoir indiquer la distance harmonique, ou relation harmonique, entre deux accords voisins. Cette relation s'exprime simplement comme l'intervalle qui sépare la basse fondamentale des deux accords ; on peut ainsi décrire deux accords comme étant en relation harmonique d'unisson (ou d'octave), de seconde ascendante (ou, par inversion, de septième descendante), de tierce ascendante (ou sixte descendante), de quarte ascendante (ou quinte descendante), et ainsi de suite.

Nous avons noté la particularité suivante des relations harmoniques entre accords de trois sons à l'intérieur d'un système diatonique : toutes les relations de quarte et de quinte, qu'elles soient ascendantes ou descendantes, comportent une note commune aux deux accords. De même, toutes les relations de tierce et 
de sixte, majeures ou mineures, ascendantes ou descendantes, comprennent deux notes communes ; les relations de seconde et de septième, quant à elles, n'en contiennent aucune. Une deuxième classification, plus simple, est donc possible, qui réduit le nombre de relations harmoniques à quatre, selon le nombre de notes communes entre deux accords.

Les relations que nous avons décrites jusqu'à présent opéraient à l'intérieur d'une tonalité fixe ; nous croyons néanmoins que cette manière de décrire les relations harmoniques demeure valable même lorsque des notes altérées surviennent dans une œuvre. C'est toujours le mouvement de la basse fondamentale qui détermine la relation harmonique ; les notes communes se trouvent souvent colorées par une altération chromatique mais n'en gardent pas moins la même appellation alphabétique.

Dans l'analyse exposée au point III du présent article, nous utilisons les abréviations suivantes : les relations de quarte et de quinte, rapport 4 ; les relations de tierce et de sixte, rapport 3 ; les relations de seconde et de septième, rapport 2 ; et les rapports d'unisson ou d'octave, rapport 1.

\section{La réverbération}

L'orgue, à l'origine un instrument hautement profane destiné à orner les jeux d'une Rome décadente, a connu son essor véritable au Moyen-âge comme instrument d'église. C'est donc dire que l'évolution de l'instrument etl'élaboration de son répertoire se sont faites dans des lieux souvent très réverbérants, comme en témoignent la plupart des églises européennes d'avant 1700 . Nous croyons que ce fait se reflète dans l'écriture de la majorité des pièces d'orgue de la période tonale et que, en particulier, l'usage de l'harmonie révèle une compréhension (active ou passive) chez les compositeurs de certains phénomènes acoustiques que nous décrirons dans les paragraphes qui suivent.

Nous avons été guidé par deux articles récents. Le premier, de Dewey Tull Lawson (1980), illustre par des exemples musicaux l'effet d'une acoustique réverbérante sur l'exécution à l'orgue et suggère plusieurs moyens d'en tirer parti ; le deuxième, de Rasch et Plomp (1982), définit d'une manière scientifique les paramètres de l'acoustique. Nous en donnons les principales conclusions ici.

L'auditeur d'une musique jouée en plein air reçoit directement des instruments l'impression sonore de l'œuvre jouée, appelée le son direct. Par contre, lorsqu'une musique est jouée à l'intérieur d'un vaisseau quelconque, l'auditeur reçoit, en plus du son direct, le son réfléchi par les murs, le plancher, le plafond, et divers objets présents dans la pièce : le son indirect qui, du fait de la plus grande distance parcourue et de l'énergie dissipée au contact des surfaces, parvient à l'oreille de l'auditeur plus tard et à plus faible intensité que le son direct, et semble provenir d'une autre direction. Les expériences décrites par Rasch et Plomp ont démontré que la partie du son indirect arrivant à l'oreille de l'auditeur moins de $25 \mathrm{msec}$ après le son direct est perçue comme faisant partie du son 
direct ; le reste du son indirect se nomme réverbération et son intensité, de même que sa durée, dépend évidemment des conditions particulières de chaque vaisseau. Le terme écho réfère à une partie du son indirect qui semble remonter soudain en intensité et qui fait croire à une nouvelle émission sonore ; il faut donc y voir un effet indésirable de la réverbération. La force relative du son direct et du son indirect dépend de la distance séparant l'auditeur de la source sonore et du degré d'absorption du son indirect dicté par le nombre d'auditeurs présents et leur habillement. Les auteurs attribuent trois effets principaux au son indirect : une augmentation apparente de l'intensité sonore, une diminution de la définition musicale par le chevauchement temporel de certains éléments et l'impression d'espace (résonance) créée par la multidirectionalité sonore. Ils concluent en suggérant que l'équilibre atteint entre la définition et la résonance est le gage d'une « bonne acoustique ».

L'article de Lawson, quant à lui, traite des résultats sur le plan musical des effets respectifs du son direct et du son indirect. Le temps de réverbération d'un vaisseau, exprimé habituellement en secondes, représente la durée du son indirect perceptible après l'arrêt du son à la source. Les notes dont l'attaque et la terminaison sont fidèlement transmises par le son direct se prolongent en dégradé dans la réverbération, créant un voile sonore qui tend à masquer les contours précis du son direct.

Il y a également un deuxième phénomène, moins bien connu, qui est d'une importance capitale pour toute musique jouée dans un lieu réverbérant. En effet, le temps de réverbération est aussi le temps que prend le son indirect pour atteindre son niveau sonore maximal ; ainsi, un accord tenu indéfiniment, au début perçu uniquement par le biais du son direct, subit une sorte de crescendo jusqu'au moment où le son indirect atteint son apogée, c'est-à-dire à la fin du temps de réverbération. On peut ainsi considérer la réverbération comme une sorte de réservoir sonore dont le temps de remplissage, comme le temps de vidange, équivaut au temps de réverbération du vaisseau. L'article de Lawson fait aussi remarquer certains aspects de la facture d'orgue classique destinés à améliorer la perception du son direct dans un lieu réverbérant, comme par exemple la présence d'un buffet entourant les tuyaux. Le son réfléchi par un buffet dont les parois sont placées près des tuyaux sera perçu comme faisant partie du son direct, puisque, comme nous l'avons vu plus haut, la première portion du son indirect s'intègre au son direct. L'attaque prononcée de certains jeux serait également destinée à une meilleure transmission du son direct par opposition au son indirect. Selon Lawson, la presque totalité de la structure temporelle (le rythme, l'articulation et le phrasé) d'une œuvre jouée dans un vaisseau réverbérant est transmise par le seul son direct. Nous avons choisi de nuancer ces propos en séparant les éléments transmis effectivement par le son direct des éléments pouvant profiter du son indirect.

Au milieu d'une pièce, lorsque le nombre de voix, la registration et d'autres 
facteurs restent fixes, le réservoir sonore dont nous empruntons l'idée à l'article de Lawson se vide à la même vitesse qu'il se remplit ; pour connaître le contenu du réservoir et, conséquemment, le son qu'il projette, on doit faire le calcul quantitatif des notes jouées pendant le temps de remplissage. Si, par exemple, on a exécuté des gammes en valeurs égales, le son du réservoir projettera un mélange des notes jouées ; si on a exécuté des arpèges, on entendra une combinaison de ces notes, et donc un accord ; si on a exécuté (comme c'est le cas le plus fréquemment) un accord principal avec divers motifs mélodiques dans les différentes voix, on entendra l'accord coloré de notes passagères.

La conséquence principale de ce phénomène est extrêmement intéressante. Des notes de durée égale seront prolongées et accentuées de manière parfaitement égale aussi par le son indirect. Par contre, toute note d'une durée relative plus longue, en subissant l'effet de crescendo déjà décrit, sera privilégiée par le son indirect. À l'encontre du son direct, qui transmet simplement les notes jouées, le son indirect effectue une double transformation, tendant à voiler les valeurs courtes (celles dont la durée est nettement inférieure au temps de réverbération) et à privilégier les valeurs longues.

\section{La registration}

Les effets de la réverbération se font sentir dans l'exécution de toutes les pièces d'orgue jouées dans un même endroit ; cependant, il est une catégorie de pièces où la qualité du son de l'instrument s'ajoute aux phénomènes purement acoustiques : il s'agit des pièces destinées au plenum. Il est intéressant de noter que ce mélange de jeux constitue, en fait, le son par essence de l'orgue, puisque les premiers instruments ne comportaient qu'un plenum à l'exclusion de tout autre jeu.

Le plenum d'un orgue classique se compose de multiples rangs de tuyaux, de principal à l'unisson, à l'octave et à la quinte de la note jouée. Les rangs les plus aigus subissent une recoupe à l'octave inférieure une fois arrivés à une hauteurplafond d'environ un huitième de pied, créant ainsi une rechute mélodique parallèle dans la voix ayant atteint cette hauteur limite. La polarité graves/aigus du clavier se trouve donc masquée par le déplacement sonore de toutes les voix d'une écriture polyphonique vers une région médiane, sans pour autant déranger leur fonction harmonique. Seule la ligne de basse, jouée indépendamment ou non à la pédale, ne subit pas cette transposition. Ce masquage de la clarté mélodique, sans préjudice à l'harmonie, renforce l'effet semblable que nous avons imputé à la réverbération. La pièce de Bach analysée sous peu est justement destinée, d'après sa forme d'écriture, à être jouée sur le plein-jeu.

\section{Le rythme harmonique}

Nous sommes maintenant en possession de tous les éléments nécessaires pour établir notre grille d'analyse. Pour cerner le rythme harmonique d'une œuvre donnée, il faudra isoler pour étude, du côté rythmique : le mètre, ainsi que les 
accentuations qui lui donnent vie, soit ses temps forts et ses temps faibles, de même que les autres accentuations agissant au niveau de l'ensemble des voix ; du côté harmonique : isoler les accords qui marquent les temps du mètre, ainsi que les accords dont la durée relative plus longue les destine à profiter des effets de la réverbération, et établir les enchaînements harmoniques reliant ces accords les uns aux autres.

\section{Analyse du Prélude en mi mineur, BWV 548, de Bach}

Description de la grille et des critères d'analyse

La pièce que nous avons choisie permettra d'illustrer le processus d'identification du rythme harmonique selon les principes énoncés précédemment. Le Prélude sélectionné a la réputation d'être un chef-d'œuvre de l'écriture pour orgue, et, tout au long de notre enquête, nous n'avons cessé d'être frappé par la minutie et la rigueur de sa composition.

Notre analyse (voir à la toute fin) prend la forme écrite d'une partition d'orgue à trois portées - reproduction fidèle du texte publié par Griepenkerl chez Peters, en 1844 - surmontée de cinq portées supplémentaires correspondant au modèle de l'analyse-réseau. Sur les portées supplémentaires sont extraits différents paramètres musicaux du texte, scrupuleusement alignés en fonction de leur apparition réelle dans l'œuvre. Les portées $A$ et $B$ présentent des éléments rythmiques ; les portées $\mathrm{D}$ et $\mathrm{E}$, des éléments harmoniques ; entre les deux couples - et à l'image du rythme harmonique, qui se situe entre les domaines du rythme et de l'harmonie - , nous avons placé notre représentation proposée du rythme harmonique de l'œuvre.

Les critères ayant servi à l'élaboration de chaque portée sont décrits plus loin. Il est à noter que les portées $\mathrm{A}$ et $\mathrm{B}$, et, dans une large mesure, la portée $\mathrm{D}$, n'ont pas nécessité de décision analytique en ce sens qu'elles transcrivent simplement et sans équivoque des réalités sonores, même si le choix de leur contenu résulte nécessairement d'une décision de l'analyste. Les portées $\mathrm{C}$ et $\mathrm{E}$, par contre, basées sur une sélection des informations contenues dans le texte ainsi que dans les portées $\mathrm{A}, \mathrm{B}$ et $\mathrm{D}$, sont orientées vers le but recherché ; leur valeur ne peut être jugée indépendamment des critères ayant servi à les élaborer.

\section{LA PORTÉE A}

Elle correspond au mètre de la pièce : chaque temps est représenté par une note noire, la première de chaque mesure constituant le 'temps fort'. Là où une deuxième série de notes parait sur la ligne supérieure de la portée (mes. 17-18, par exemple) se manifeste un nouveau mètre, suggéré temporairement par des événements rythmiques ; ce nouveau mètre ne remplace toutefois pas le mètre original.

\section{LA PORTÉE B}

Elle présente trois fils rythmiques, extraits du tissu rythmique. Sur la ligne inférieure se trouve le rythme composite, c'est-à-dire la somme de toutes les 
attaques dans toutes les voix, en comptant une seule fois les attaques simultanées. Sur la ligne médiane sont notées les attaques simultanées de trois voix ; enfin, sur la ligne supérieure prennent place les attaques simultanées de quatre ou de cinq voix.

\section{LA PORTÉE C}

Cette portée représente le rythme harmonique de l'œuvre. Nous préférons reporter à plus tard notre explication de l'origine de cette représentation; il suffit de noter pour l'instant que les hampes de note orientées vers le bas indiquent des informations redevables au domaine harmonique, tandis que les hampes orientées vers le haut fournissent des informations issues du domaine rythmique.

\section{LA PORTÉE D}

Le phénomène de la résonance a guidé l'élaboration de cette portée. Nous avons cherché à représenter les accords les plus aptes à entrer un moment dans la composition du réservoir sonore, et donc à être perçus par le biais du son indirect. Les accords en notes blanches ont une durée de plus d'un temps, les accords en notes noires d'un temps seulement ; les accords de moins d'un temps, trop courts pour dominer le réservoir sonore, n'ont pas été considérés. Il est à remarquer qu'une note faisant partie d'un accord blanc devient elle-même blanche, peu importe sa longueur et son emplacement réels; il en va de même des notes noires faisant partie d'un accord noir. Nous avons retenu en premier lieu les notes entendues sur le premier temps d'une mesure ; si ces notes forment déjà un accord triadique de trois ou de quatre sons, nous leur imputons la résonance du réservoir sonore tant que la majorité des notes suivantes (prioritairement celles entendues sur un temps, fort ou faible) font encore partie du même accord. Si le premier temps fait entendre un accord dont une note est retardée par la marche du contrepoint, nous indiquons l'accord avec les moments d'arrivée des notes constituantes. Nous fixons les changements d'accord soit d'après l'arrivée d'un nouveau temps fort, soit d'après la coïncidence de trois notes sur un autre temps formant sans équivoque un nouvel accord d'une durée d'au moins un temps.

\section{LA PORTÉE E}

Cette portée donne la basse fondamentale des accords dont l'attaque est notée à la portée $\mathrm{C}$. Chaque chiffre apparaissant au-dessus de la portée indique la relation harmonique des deux accords placés de part et d'autre de ce chiffre : en caractères gras, les relations harmoniques qui introduisent le premier temps d'une mesure, et, en caractères ordinaires, les relations harmoniques entre deux accords à l'intérieur d'une mesure. Les valeurs de notes de cette ligne analytique, non présente dans l'œuvre, ne sont pas exactes : les notes noires et les notes blanches dérivent de la portée $\mathrm{D}$; les notes en forme de croche, de la portée $\mathrm{C}$.

\section{Résultats de l'analyse}

Nous allons décrire les résultats obtenus en considérant séparément les cinq portées, dans l'ordre suivant : B, A, D, C, et E. C'est en effet la portée B, celle 
relevant la simultanéité des attaques, qui a fourni les premiers éléments pour l'interprétation des autres portées.

\section{portée $B$}

Cette deuxième portée présente trois fils rythmiques extraits du Prélude de Bach. Parmi la multitude des séries d'accentuations que contient l'œuvre, nous avons choisi d'étudier celles qui proviennent d'attaques simultanées dans plusieurs voix, en suivant l'indice fourni par les mesures initiales: on retrouve en effet dans la toute première mesure une attaque simultanée de cinq voix (y compris la pédale), une attaque de pédale seule, cinq attaques de voix mélodique seule et une attaque de quatre voix manuelles sans pédale. Déjà, une texture aussi peu homogène laisse présager l'importance accordée par le compositeur à cet aspect de l'écriture instrumentale.

Une fois prise la décision de considérer la simultanéité des attaques, il nous a fallu effectuer une sélection, car le nombre de simultanéités possibles est considérable : les attaques à une, deux, trois, quatre et cinq voix, avec et sans pédale, entre parties voisines ou distancées, et ainsi de suite. Nous avons regroupé certaines catégories et délaisé certaines autres pour retenir les suivantes : les attaques simultanées de quatre ou de cinq voix, avec ou sans pédale ; les attaques de trois voix, avec ou sans pédale ; et le rythme composite, ou somme des attaques.

En parcourant la ligne inférieure de la portée $B$, celle du rythme composite, une première constatation s'impose : un rythme de doubles croches continues est omniprésent, sauf dans les mesures où est présentée la première idée mélodique (mes. 1-5, 33-37, 81-85, 86-90), dans des sections basées sur la tête de cette idée (59-60, 70-75) et dans deux mesures isolées (12-13) reprises à la dominante (44-45) ainsi qu'à la fin du mouvement (130-131). On voit ainsi le retour d'un élément thématique signalé par un changement sensible dans le rythme composite.

La ligne supérieure de la portée $B$ répertorie les attaques simultanées de quatre ou de cinq voix. Au cours du mouvement, ces attaques surviennent, à quelques exceptions notables près, à la même conjoncture métrique : Bach écrit deux attaques à une croche de distance, à cheval sur la barre de mesure.

Les exceptions à cet emplacement des attaques à quatre ou à cinq voix aux changements de mesure sont de deux ordres. Premièrement, des attaques solitaires, placées sur le premier temps d'une mesure après des enchaînements harmoniques rapides (mes. 46-48 et 51). Deuxièmement, les attaques marquant les cadences des grandes sections de l'œuvre. Ainsi, à la mesure 18, les deux attaques successives, entendues auparavant comme levée et battue du premier temps d'une mesure, remplissent ici la même fonction au deuxième temps, déplaçant le temps fort et créant l'hémiole. Aux mesures 16-17, la multiplication des attaques signale l'arrivée prochaine de la cadence ; à la mesure 19, une attaque solitaire sur le premier temps rétablit l'ordre bousculé par l'hémiole. 
Les autres grandes cadences sont marquées par des techniques semblables, mais les attaques à trois voix remplacent souvent les attaques à quatre et cinq voix utilisées pour la première cadence. À certains endroits, il existe un véritable dialogue entre les attaques à trois voix d'une part, et les attaques à quatre ou à cinq voix d'autre part, comme par exemple aux mesures 12 à 19 ou 44 à 51 .

\section{portée A}

Dès la première mesure de la pièce, la hiérarchie rythmique s'établit de façon claire, les pulsations des niveaux rapides (doubles croches et croches) étant groupées par deux, et la pulsation de noires, fournie par les accords tenus et les notes réitérées à la pédale, donnant lieu à des groupes de trois. Nous percevons ainsi le mètre de l'œuvre : une pulsation régulière de noires, groupées en trois par l'action de l'harmonie. Une fois établi, ce mètre est le plus souvent confirmé par les événements harmoniques, mais son fonctionnement autonome lui permet de définir à son tour le rôle de certaines harmonies.

Nous avons noté à quatre endroits une perturbation majeure du mètre : aux mesures $17-18,49-50,79-80$ et 136-137. Ces endroits correspondent à des cadences définissant les grandes sections de l'œuvre et, comme nous l'avons vu plus haut, les événements rythmiques y créent l'hémiole en transférant temporairement l'accent du premier temps de la mesure vers le deuxième ou le troisième temps. Deux autres cadences, mesures 31-32 et 101-102, ne présentent pas d'hémiole à proprement parler, mais plutôt une accumulation d'accents qui donne autant d'importance au deuxième temps de la mesure qu'au premier.

Dans deux autres sections de l'œuvre, mesures 103-108 et 116-119, nous avons noté sur la portée A une perturbation possible du mètre ressemblant à l'hémiole. Notre interprétation de ces passages se base essentiellement sur des facteurs harmoniques que nous présenterons plus loin.

La hiérarchie rythmique du Prélude en mi mineur présentée dès les premières mesures laisse entrevoir un niveau métrique supérieur à la mesure, c'est-à-dire un niveau qui regroupe les mesures entre elles. L'harmonie de 'mi', répétée à la deuxième mesure, permet en effet de postuler un groupement des mesures par deux, groupement qui semble confirmé et renforcé par les changements de texture et de matériau thématique dans les mesures suivantes. On peut suivre ce système jusqu'au retour du matériau thématique principal, maintenant à la dominante, à la mesure 33 , et même jusqu'à sa troisième apparition à la mesure 81. À la mesure 86, cependant, le groupement est rompu par l'entrée du même matériau à la sous-dominante, sur une mesure paire.

Cette analyse sommaire confirme une notion que nous avons retenue plus haut dans le travail de Lester, notion selon laquelle les groupements métriques effectués à un niveau supérieur à la mesure paraissent de façon intermittente au cours d'une œuvre, cédant devant une organisation plus organique des phrases musicales. 
portée $D$

Les critères gouvernant la formulation de la portée $\mathrm{D}$ ont été décrits plus haut. Notre première tentative de ne considérer que les notes possédant une durée matériellement plus longue que les autres, sans rapport avec le mètre ni avec une conception harmonique quelconque, s'est soldée par l'échec. Certaines notes essentielles à l'harmonie n'ont qu'une durée réelle très courte ; et il arrive que des notes étrangères aient un poids temporel important. En fait, la clef de cette sélection a été la même qu'utilise l'auditeur devant l'infini mélange de sons qui l'assaille : le mètre. Avec sa succession régulière de temps forts et de temps faibles, le mètre offre la trame nécessaire pour distinguer les mélanges structurels des mélanges passagers.

Parcourir visuellement la portée D équivaut en quelque sorte à entendre l'œuvre dans un lieu très réverbérant, où l'on se trouve placé de telle sorte que le son direct ne nous atteint pas. On remarquera que certaines harmonies sont clairement énoncées et bien espacées tandis que d'autres se suivent rapidement, leurs notes constituantes semblant même se chevaucher. L'alignement vertical des notes sur la page fournit l'image de leur présentation temporelle : on entend toutes les notes d'un accord en même temps, ou bien arpégées, ou encore, certaines notes se font attendre par les artifices de l'écriture, les retards, les appoggiatures, les notes de passage accentuées. Puisque nous n'avons capté sur cette portée que les accords d'une durée d'au moins un temps, certaines parties du discours disparaissent complètement : ce sont les éléments véhiculés d'une façon privilégiée par le son direct, tel le dessin mélodique au soprano dans les premières mesures de l'œuvre.

\section{portée $C$}

Loin de la rigueur cartésienne des portées $\mathrm{A}$ et $\mathrm{B}$, la portée $\mathrm{C}$ offre une proposition seulement de ce qu'on pourrait appeler le rythme harmonique de l'œuvre étudiée. La sélection et l'usage que nous faisons des éléments concrets extraits du discours demeurent subjectifs ; il se peut que ce résultat reflète plus les volontés de l'analyste qu'une réalité sonore. Nous donnerons ici les raisons qui nous ont amené à faire certains choix. Redisons que la portée $\mathrm{C}$ a été placée symboliquement entre les portées représentant l'activité rythmique de l'œuvre et celles résumant les harmonies marquantes.

Le terme 'rythme harmonique' veut dire essentiellement : un rythme dessiné dans le temps par certaines harmonies d'une pièce. Le travail d'analyse que nous avons effectué n'avait pour but que de nous permettre de faire un choix éclairé des harmonies significatives sur le plan rythmique. Il nous a paru primordial d'inclure dans la portée $C$ les harmonies dont la longueur relative et l'emplacement métrique en font le contenu du réservoir sonore ; mais n'inclure que ces harmonies donnait un résultat tristement carré qui semblait trahir l'intense activité rythmique de l'œuvre. Nous nous sommes alors tourné vers les événements marquants que sont les attaques simultanées à plusieurs voix : ces attaques 
peuvent-elles signaler certaines harmonies délaissées dans la portée $D$ à cause de leur peu de durée ? En outre, de même que la portée D représente les éléments susceptibles d'être perçus par le biais du son indirect, est-ce que certains accords peuvent être véhiculés par le son direct moyennant une technique pour les porter à l'attention de l'auditeur ? C'est en répondant oui à ces questions que nous proposons la lecture de la portée $\mathrm{C}$.

Notre méthode a consisté à inclure sur la portée du rythme harmonique : premièrement, les accords d'une durée d'un temps ou plus, déjà répertoriés sur la portée $\mathrm{D}$; deuxièmement, les accords signalés par une attaque simultanée de quatre ou de cinq voix ; et troisièmement, tout accord provenant d'une attaque simultanée de trois voix, mais dans les seuls cas où cet accord différait de l'accord le précédant ou, encore, apportait un élément nouveau à ce dernier (par exemple, l'ajout d'une septième). Dans certains cas, des attaques simultanées ont permis de restituer le début d'un accord mal défini dans le son indirect (par exemple, aux mesures 21-23).

L'écriture de la portée $\mathrm{C}$ rend donc compte de la provenance des accords : la hampe de note orientée vers le bas indique un accord présent dans le son indirect, celle orientée vers le haut, un accord signalé par le son direct.

\section{portée $E$}

Nous avons noté à la portée E les basses fondamentales des accords retenus pour faire partie du rythme harmonique et dont les attaques figurent à la portée $\mathrm{C}$; les valeurs sont approximatives et indiquent la provenance de l'accord en question, soit de la portée $\mathrm{D}$, soit de la portée $\mathrm{B}$. Les chiffres placés au-dessus de la portée indiquent la relation harmonique entre deux accords, selon le système proposé antérieurement, les chiffres en caractères gras se rapportant à la relation qui introduit le premier accord d'une mesure.

Toute tentative de chiffrage dans le domaine harmonique comporte nécessairement une marge d'interprétation. Ainsi, en cherchant à donner une basse fondamentale à chacun des accords choisis pour figurer à la portée $\mathrm{C}$ de notre analyse, nous nous sommes heurté à un problème qui a sollicité des solutions diverses au cours des siècles (Konecne 1989). En effet, s'il est relativement facile de repérer des accords formés uniquement d'intervalles consonants et de leur attribuer une basse fondamentale, la présence d'un intervalle dissonant peut renvoyer, selon l'interprétation, à deux basses fondamentales diférentes. Et que faire d'un accord contenant, par exemple, un double retard ou une double appoggiature à résolution obligée, mais qui a en lui-même l'apparence d'un accord consonant ou dissonant sur une basse fondamentale différente ? Nous avons adopté la solution suivante : en accord avec notre désir de n'effectuer aucune manipulation temporelle, nous avons chiffré les accords selon leur apparence simple, sans tenir compte de leur résolution éventuelle. Les enchaînements sur pédale sont également chiffrés en faisant abstraction de la note de pédale. Cette attitude 
scolastique, à l'encontre des techniques de réduction employées couramment, permet de dégager les relations harmoniques au niveau le plus élémentaire ; les endroits où cette manière de chiffrer se prête le plus à discussion sont aussi riches de renseignements et seront étudiés plus loin.

Nous avons pu constater dans le Prélude en mi mineur un usage systématique des différents types de relations harmoniques, et nous croyons que l'identification de certains de ces types permet à l'auditeur, à défaut d'autres moyens, de placer le temps fort d'une mesure par rapport aux temps faibles.

Notre classification distingue quatre types de relation harmonique selon la distance séparant les deux basses fondamentales et le nombre de notes communes entre deux accords de trois sons. Ainsi, le rapport 1 décrit la relation entre deux accords identiques ou bien deux renversements du même accord ; toutes les notes sont alors communes entre ces deux accords. Le rapport 2 décrit la relation entre deux accords dont les basses fondamentales sont séparées par une seconde ou, par renversement, par une septième ; il n'y a alors aucune note commune. Le rapport 3 décrit la relation entre deux accords dont les basses fondamentales sont distantes d'une tierce ou d'une sixte ; deux notes sur trois sont communes aux deux accords. Finalement, le rapport 4 désigne la relation entre deux accords dont les basses fondamentales sont séparées d'une quarte ou d'une quinte ; il y a une note commune sur trois. Ce rapport 4 , sorte de juste milieu entre l'identification trop proche des accords en rapport de tierce et l'absence de notes communes des accords en rapport de seconde, est la base de la syntaxe harmonique classique.

Dans la basse fondamentale que nous avons élaborée à partir des accords jugés essentiels dans le Prélude en mi mineur, le rapport 1, bien que fréquent à l'intérieur d'une mesure, ne se présente qu'à un seul endroit dans le passage d'une mesure à une autre. Les rapports 2 et 4 sont aussi fréquents entre les mesures qu'à l'intérieur de celles-ci. Quant aux rapports 3, leur emploi se limite aux intérieurs de mesure, sauf à deux endroits bien délimités.

Nous avons déjà eu l'occasion d'étudier les cadences marquant la fin des principales sections de l'œuvre. À la quatrième de ces cadences, mesures 79-80, nous retrouvons le premier emploi du rapport 3 entre deux mesures : l'accord sur 'sol' du troisième temps de la mesure 79 s'enchaînant à l'accord sur 'mi' du premier temps de la mesure 80 . On contate, d'après les notes isolées sur la portée D, le rapprochement de ces deux accords par les notes communes ; en fait, l'accord de septième sur 'mi' contient déjà les trois notes de l'accord simple sur 'sol'. Nous croyons que l'emploi du rapport 3 à ce moment précis fait partie de l'ensemble des techniques employées pour affaiblir le premier temps en transférant son poids métrique vers le deuxième temps, ensemble connu sous le nom d'hémiole. Sous cet angle de vue, les points d'accentuation de la portée B, en plus de transférer un dessin rythmique caractéristique du premier temps sur le deuxième temps de la mesure, servent aussi à mettre en relief un rapport harmonique qui concourt au même but. 
Le rapprochement par notes communes que nous venons de remarquer entre les accords de 'mi' et de 'sol' (mes. 79-80) vaut aussi pour la relation 3 entre ce même accord de 'mi' et l' accord suivant, un accord de 'do'. Par cet enchaînement de relations de tierce, l'accord de 'sol' du dernier temps de la mesure 79 peut être considéré non pas comme accord de tonique dans la tonalité de sol, mais plutôt comme accord faisant partie du complexe étendu de la sous- dominante.

Les mesures 103-108 du Prélude font paraître un usage plus étendu des rapports 3 entre mesures. Sous l'apparition régulière d'un motif en imitation entre deux voix qui se conforme au mètre écrit, le jeu des harmonies fait entendre un rappport 3 sur toutes les deux barres de mesure. À ces endroits, le rapprochement des accords par notes communes diminue le poids métrique attendu sur le premier temps, ce poids étant plutôt attribué au deuxième temps introduit par un rapport 4. Le mètre est moins manifestement ébranlé par ce procédé que par l'hémiole étudiée précédemment ; il s'agit, à notre avis, d'une subtilité de l'écriture faisant coexister pendant ces six mesures deux interprétations métriques parallèles. Il est intéressant de constater que la notation de la réinterprétation métrique de la portée $\mathrm{A}$, la même que nous avons utilisée pour indiquer l'hémiole, regroupe les mesures par deux en commençant par une mesure impaire, et se conforme ainsi au groupement par deux manifesté au début de l'œuvre.

Aux mesures 116-119, une autre série de motifs provoque l'affaiblissement du poids métrique du premier temps. L'enchaînement des mesures 116 à 117 et 118 à 119 fait entendre deux accords de trois sons qui peuvent s'analyser comme un seul accord de septième. Selon notre système de classification des relations harmoniques, c'est là le seul moment de la pièce où un rapport 1 est utilisé entre deux mesures. Le groupement temporaire par deux mesures, indiqué à la portée A, commence ici par une mesure paire et ne se conforme donc pas au groupement esquissé dans les premières mesures.

Il se trouve quelques endroits dans le Prélude en mi mineur où la basse fondamentale que nous avons indiquée et les relations harmoniques qui en résultent sembleront équivoques ou non fondées. Par exemple, dans les mesures 21-24, le premier temps de chaque mesure est marqué par un retard au soprano dont la résolution devient retard à la mesure suivante, mettant en relief un dessin mélodique descendant du 'sol' de la mesure 21 au 'si' de la mesure 27, repris immédiatement en sens inverse jusqu'à la mesure 32. Or, c'est pour éviter la réduction temporelle que nous avons identifié l'accord intervenant au premier temps de la mesure 22 comme un accord de neuvième sur basse fondamentale 'fa ', même si sa résolution éventuelle démontre plutôt un accord de sixte sur basse fondamentale 'ré', avec double retard. Nous croyons que l'important, ici, n'est pas de trancher pour l'une ou l'autre de ces deux interprétations possibles, mais de constater plutôt que l'ambiguiité se situe à une différence de tierce entre les deux basses fondamentales et donc, que la distinction faite entre les relations 'fortes' 2 et 4 à la barre de mesure et les relations 'faibles' 3 et 1 à l'intérieur de 
la mesure se maintient, peu importe la solution choisie. Il en va de même aux mesures 51-54, ainsi qu'à d'autres endroits où le rapprochement par notes communes rend difficile l'identification de la basse fondamentale.

\section{Conclusions}

Afin de situer l'aire d'action de cette notion vague qu'était, au début de nos recherches, le rythme harmonique, notre étude du Prélude en mi mineur aura nécessité la mise en place d'une grille d'analyse adaptée ainsi qu'un examen attentif de l'interaction du rythme avec l'harmonie. Du côté rythmique, nous avons essayé de cerner le fonctionnement du mètre et, surtout, d'expliquer l'importance, dans une œuvre tonale, des séries d'accentuation créées par des paramètres qu'il est possible d'isoler, tels les sommets mélodiques, les attaques, les changements de texture et ainsi de suite. Du côté harmonique, nous avons cherché les raisons acoustiques qui mettent à l'avant certains accords parmi les multiples rencontres harmoniques d'une pièce tonale ; nous avons également examiné les relations harmoniques qui appuient le mètre et les autres liens entre harmonie et mètre.

Le rythme harmonique constitue, croyons-nous, une de ces séries d'accentuation rythmique que nous avons appelées fils rythmiques, mais ses éléments propres sont certes plus difficiles à isoler que, par exemple, les attaques simultanées ou les débuts de motif. Chaque accord retenu dans notre esquisse du rythme harmonique a nécessité une justification basée sur des paramètres eux-mêmes extraits du texte musical selon des critères fixes, ces paramètres étant le mètre, la résonance et les attaques simultanées à plusieurs voix. C'est donc dire que notre rythme harmonique représente une double sélection de la réalité sonore ; l'origine de nos critères d'analyse importe autant que leur emploi. Ce que nous avons proposé est, en fait, non pas le rythme harmonique de la pièce, mais un rythme harmonique possible, justifié par les critères que nous avons retenus ; un changement de ces critères amènerait fatalement un changement du rythme harmonique proposé.

Il y a cependant un élément de contrôle dans notre analyse qui l'empêche de devenir une pure abstraction : nous n'avons fait que privilégier des éléments déjà présents dans la partition, sans les transformer ni les déplacer. La gamme des résultats éventuels va donc d'une portée vierge, où aucun accord du texte musical n'aurait rempli les critères fixés, à une portée reproduisant intégralement le texte de Bach, où la multiplicité des critères aurait permis l'inclusion de chaque note du Prélude. Notre approche se situe entre ces deux pôles.

Nous croyons que l'interdépendance du mètre avec le schéma harmonique justifie l'inclusion dans le rythme harmonique des accords marquants et marqués par le temps fort, d'autant plus que ces accords sont souvent ceux qui, par leur durée, dominent l'effet du réservoir sonore. Nous y voyons le premier niveau du rythme harmonique. Le deuxième niveau ajoute les accords nouveaux (différents 
des accords du premier temps) des temps faibles et les accords susceptibles de paraître, par leur durée, dans le réservoir sonore. Pour ce qui concerne le troisième niveau, il inclut les accords signalés par des attaques simultanées à plusieurs voix, attaques que nous avons séparées en deux catégories; un raffinement de ces catégories (qui consisterait, par exemple, à distinguer les attaques avec pédale des attaques sans pédale) ne nous semblait pas apporter un raffinement correspondant du rythme harmonique, même si cela pouvait s'avérer significatif sur le plan de la différenciation rythmique des thèmes.

Notre démarche a donné le résultat indiqué à la portée $\mathrm{C}$ de l'analyse. De par la variété des dessins rythmiques proposés (souvent continus sur plusieurs mesures) et leur concordance entre sections de matériau thématique similaire, nous croyons que le rythme de la portée $\mathrm{C}$, réuni à ses notes de basse véritables et fourni d'accords dans la manière d'un continuo, ne trahirait pas le déroulement rythmique et harmonique du Prélude en mi mineur. Nous avons été frappé par la diversité des configurations de la portée $\mathrm{C}$, ses plages de stabilité, d'agitation, de disparité avec le mètre noté, et sa façon de s'alimenter tour à tour du domaine harmonique et du domaine rythmique.

Sur un point précis, nous sommes en désaccord avec la définition courante du rythme harmonique telle qu'elle a été précisée au début de cette étude. Car, ayant noté à plusieurs endroits l'importance accordée à la réitération d'un même accord, nous pensons que certains accords gardent une importance structurelle même s'ils n'introduisent pas une nouvelle basse fondamentale; en fait, dans un contexte général procédant par changements d'accords, la réitération constitue un moyen efficace de mettre en relief un accord donné.

Notre choix du Prélude en mi mineur comme objet d'investigation n'a pas été entièrement innocent ; la perfection de son écriture, sa construction rigoureuse et l'inépuisable variété de ses textures nous promettaient à l'avance une analyse cohérente. Le but de notre travail était cependant plus large : faire, d'une part, la démonstration du fonctionnement du rythme harmonique et élaborer, d'autre part, un système permettant à l'interprète de saisir l'essence du rythme harmonique de toute pièce tonale. La grille d'analyse que nous avons présentée ici pourra-telle s'appliquer à d'autres œuvres ? Nous croyons que oui ; les étapes de notre travail sont suffisamment distinctes pour permettre leur adaptation ou leur remplacement par d'autres étapes, selon la pièce à étudier. Les éléments servant à identifier le troisième niveau du rythme harmonique devront en particulier être choisis avec soin en fonction de l'écriture. Dans le cas de l'orgue, où aucune différenciation dynamique n'est possible, les attaques simultanées constituent probablement une technique répandue pour souligner certains événements du discours musical ; des analyses futures d'œuvres de catégories et de compositeurs variés permettront de regrouper une série de techniques d'écriture pouvant servir à marquer et donc, pour l'analyste, à déceler le rythme harmonique.

D'autres pistes pour l' avenir nous sont apparues au cours de ce travail. L'analyse 
selon le même système de plusieurs œuvres du répertoire permettrait ensuite d'effectuer une comparaison stylistique pour dégager des techniques courantes de manipulation du rythme harmonique. La coïncidence relative du système consonance/dissonance avec le système temps fort/temps faible du mètre au cours d'une pièce nous semble un facteur important de tension et de détente qui mériterait étude. L'analyse de la relation harmonique entre accords, démontrant l'usage de certains enchaînements pour indiquer le temps fort et, par conséquent, le mètre, a constitué l'un des aspects de cet article ; l'évolution de ces usages et particulièrement l'affranchissement du rapport de tierce, altéré ou non, au cours du XIX ${ }^{\mathrm{e}}$ siècle, nous paraissent également dignes d'attention.

Le rythme harmonique, en lien étroit avec le mètre, représente certainement un facteur d'organisation important dans le langage de la musique tonale. Et nous croyons fermement que ce genre d'information obtenue par l'analyse peut être d'une utilité directe pour l'interprète.

BENJAMIN, William E.

\section{RÉFÉRENCES}

1984 : «A Theory of Musical Meter », Music Perception, 1/4 : 355-413.

BENT, Ian et DRABKIN, William

1987 : Analysis. New York : Norton.

BARRY, Walter

1985 : « Metric and Rhythmic Articulation in Music », Music Theory Spectrum, VII : 7-33.

CAPLIN, William E.

1980 : " Harmony and Meter in the Theories of Simon Sechter », Music Theory Spectrum, II : 74-89.

1983 : «Tonal Function and Metrical Accent : A Historical Perspective », Music Theory Spectrum, V : 1-14.

FORTE, Allen

1962 : Tonal Harmony in Concept and Practice. New York : Holt, Rinehart and Winston.

GJERDINGEN, Robert O.

1988 : A Classic Turn of Phrase : Music and the Psychology of Convention. Philadelphia : University of Pennsylvania Press.

GOLDMAN, Richard F.

1965 : Harmony in Western Music. New York : Norton.

KONECNE, Julie L.

1989: «Harmonic Reduction Techniques in Music Analysis, c. 1770-1917», Ph. D. diss., University of Iowa. 
KRAMER, Jonathan D.

1985: «Studies of Time and Music: A Bibliography », Music Theory Spectrum, VII : 72-106.

LAWSON, Dewey T.

1980 : "Church Acoustics : Implications for Organ Performance ", The Organ Yearbook, XI : 116-120.

LESTER, Joel

1986: The Rhythms of Tonal Music. Carbondale and Edwardsville : Southern Illinois University Press.

MARKS, James B.

1969: « Harmonic Rhythm as a Factor in Form Delineation in Selected Instrumental Works from 1775 to 1850 », Ph. D. diss., Indiana University.

NARMOUR, Eugene

1977 : Beyond Schenkerism : The Need for Alternatives in Music Analysis. Chicago : The University of Chicago Press.

NORTON, Richard

1984 : Tonality in Western Culture : A Critical and Historical Perspective. University Park : The Pennsylvania State University Press.

RASCH, R.A. et PLOMP, R.

1982: «The Listener and the Acoustic Environment », The Psychology of Music, Diana Deutsch éd., 135-146. New York: Academic Press.

SADAI, Yizhak

1980: Harmony in its Systemic and Phenomenological Aspects. Jerusalem: Yanetz, 1980.

SCHENBERG, Arnold

1969 : Structural Functions of Harmony. New York : Norton.

TALBOT, Michael

1972: «Harmony and Meter : A Study in Relationships», The Music Review, $23: 47-52$.

WESTERGAARD, Peter

1975 : An Introduction to Tonal Theory. New York : Norton.

YESTON, Maury éd.

1977: Readings in Schenker Analysis and Other Approaches. New Haven : Yale University Press. 
-I-

A
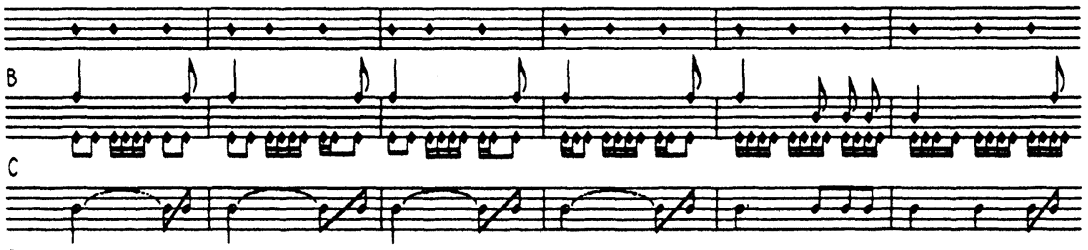

0
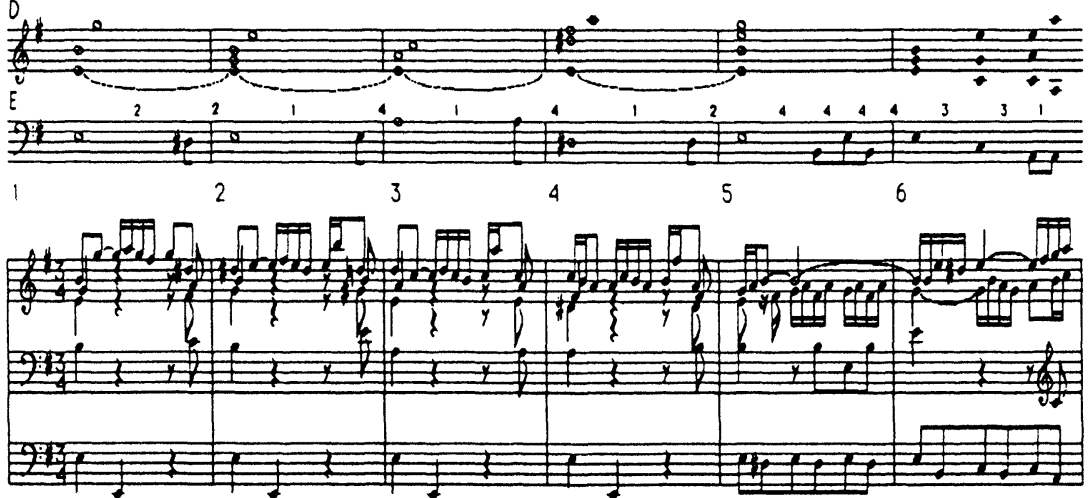

A

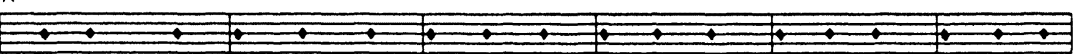
(LI $C$

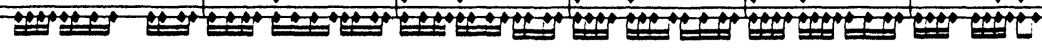
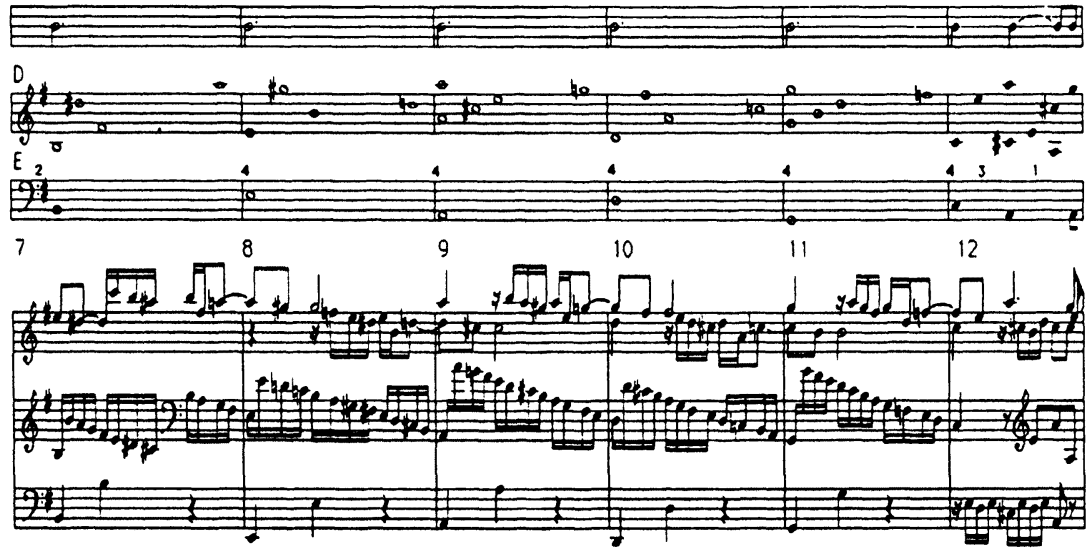
-II-

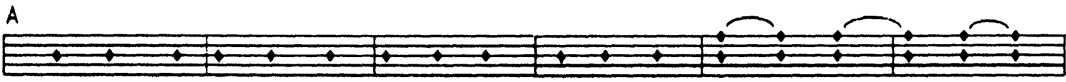

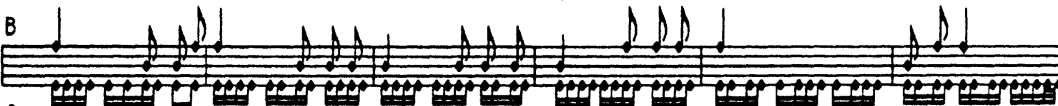
C

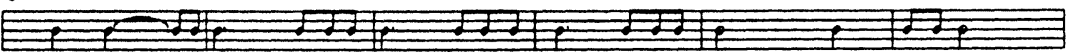

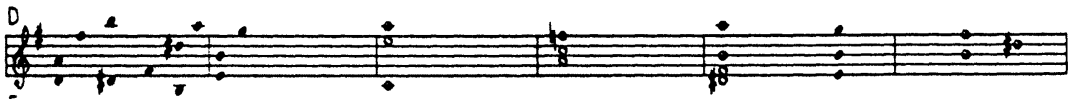

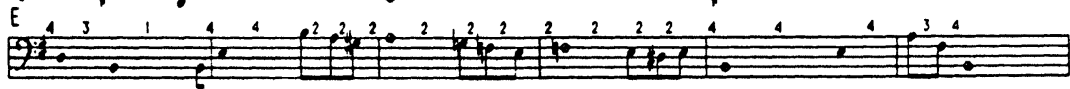

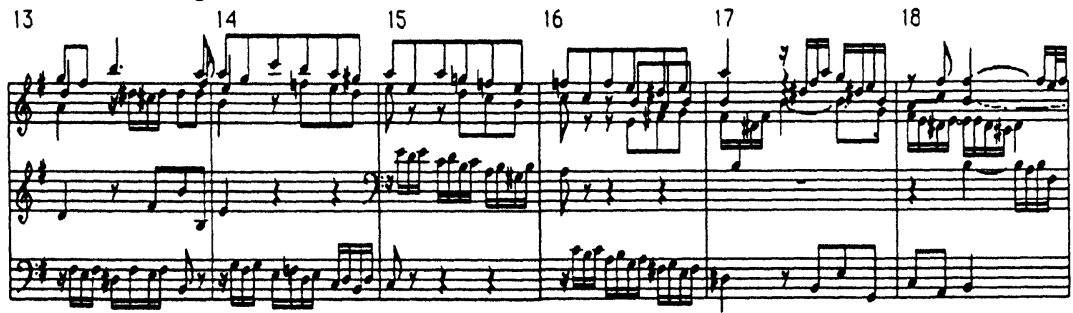

A

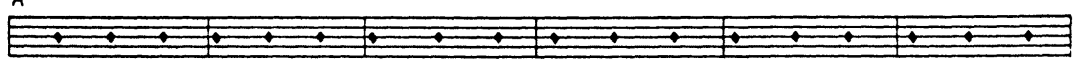
LL+ C

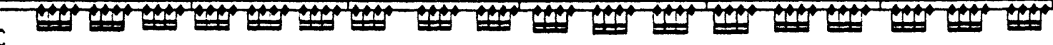

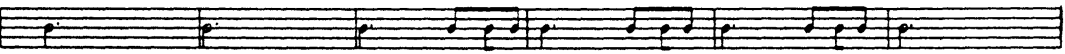
$80^{102}$ E: 4
19
20
21
22
23
24

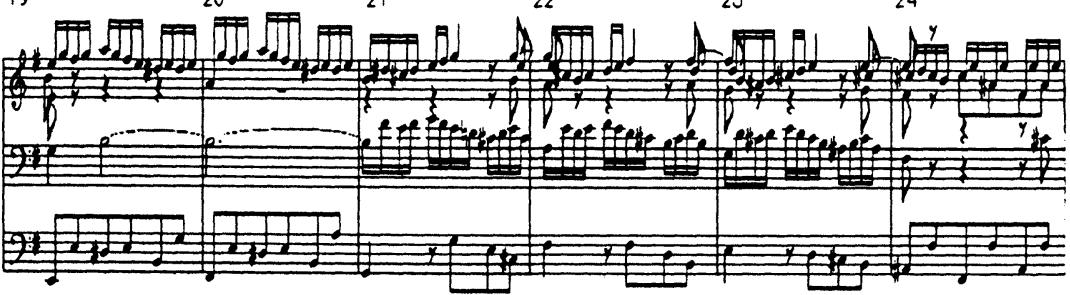


A
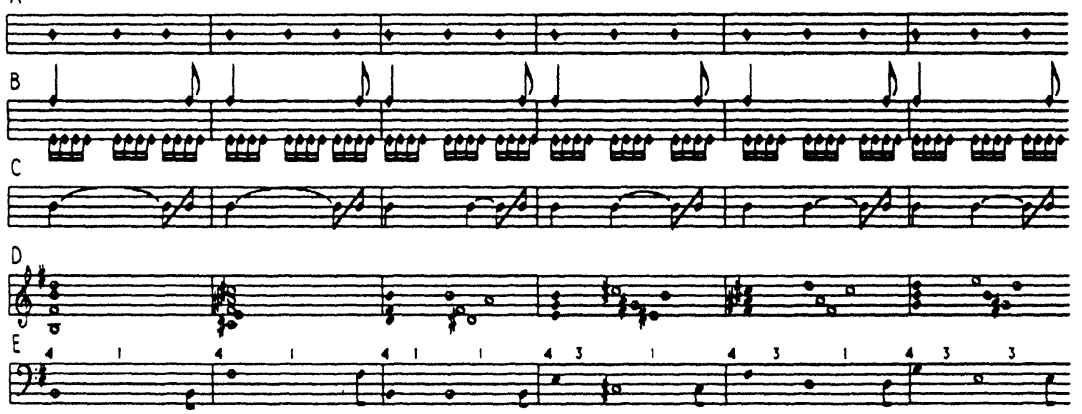

25

26

27

28

29

30

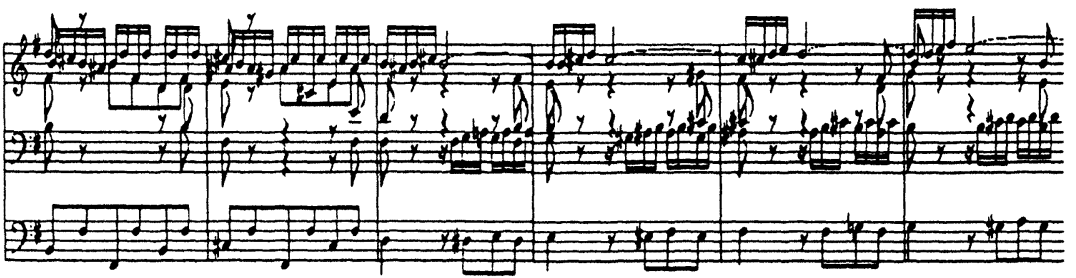

A

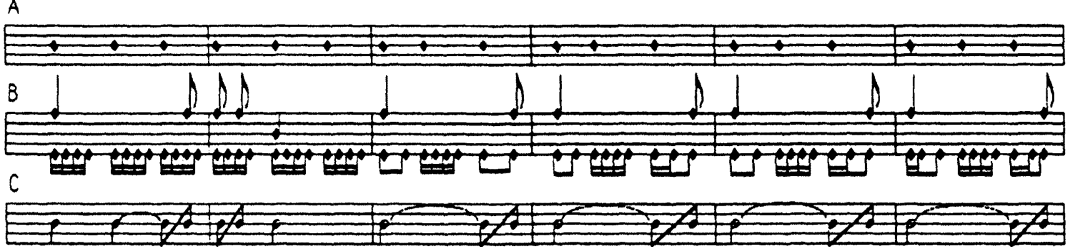

W .....
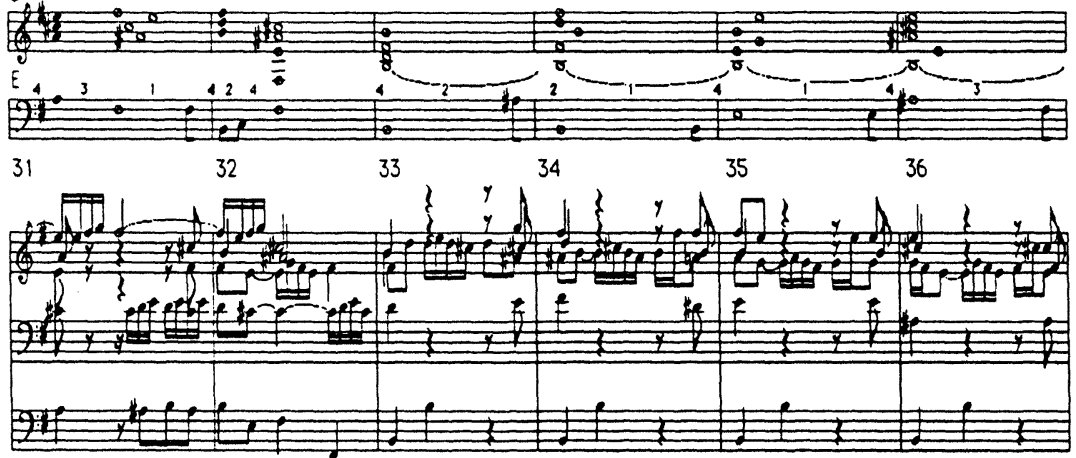
A

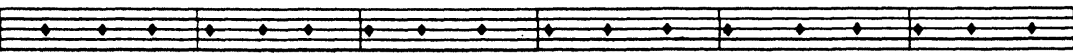
50

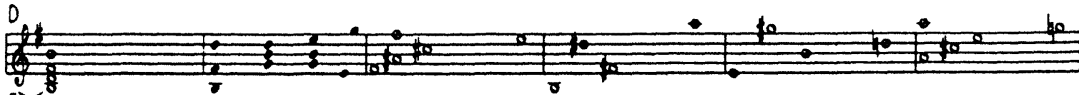

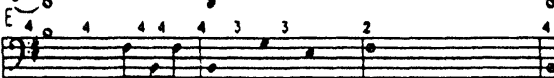

(37)
(1) 
-V.

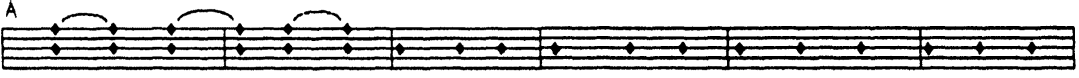
(1) 20 (29)

A $\Longrightarrow \square$

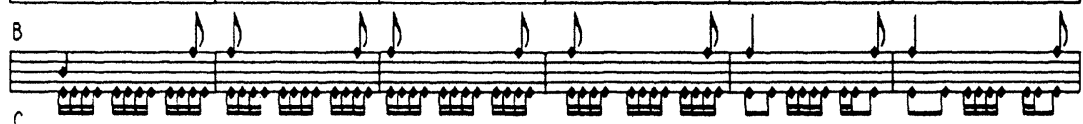

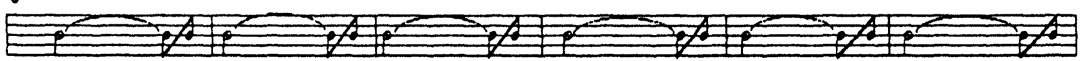

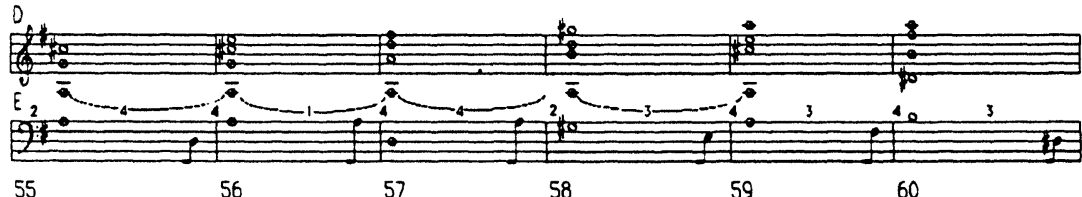

$\begin{array}{llllll}55 & 56 & 57 & 58 & 59 & 60\end{array}$

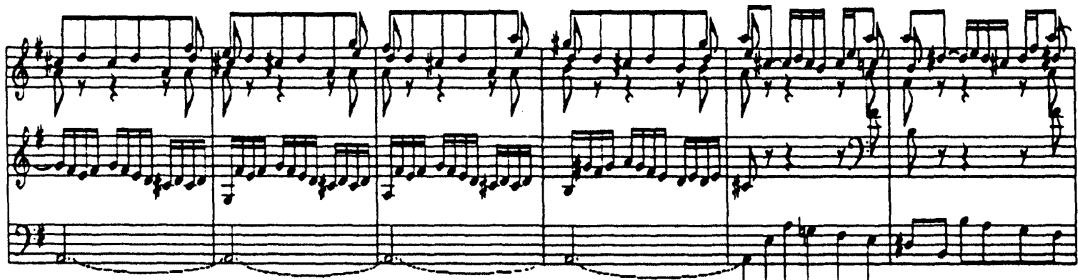


A
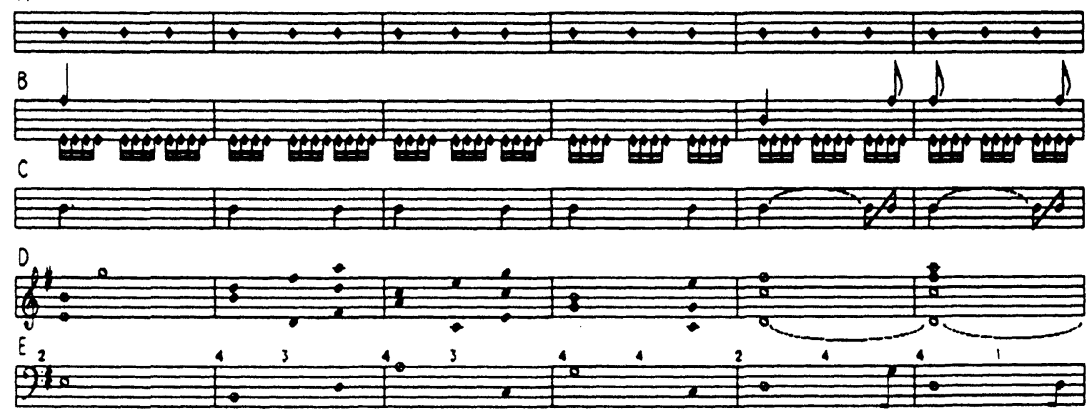

(15)

A

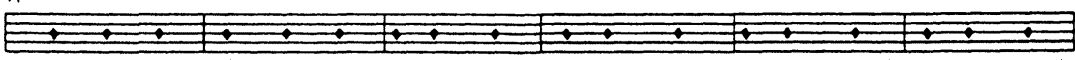

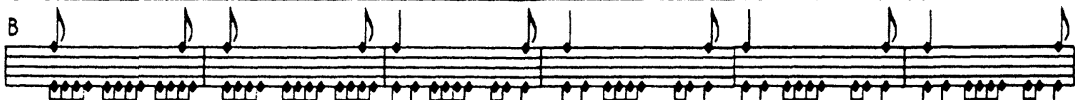
C

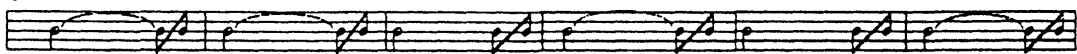
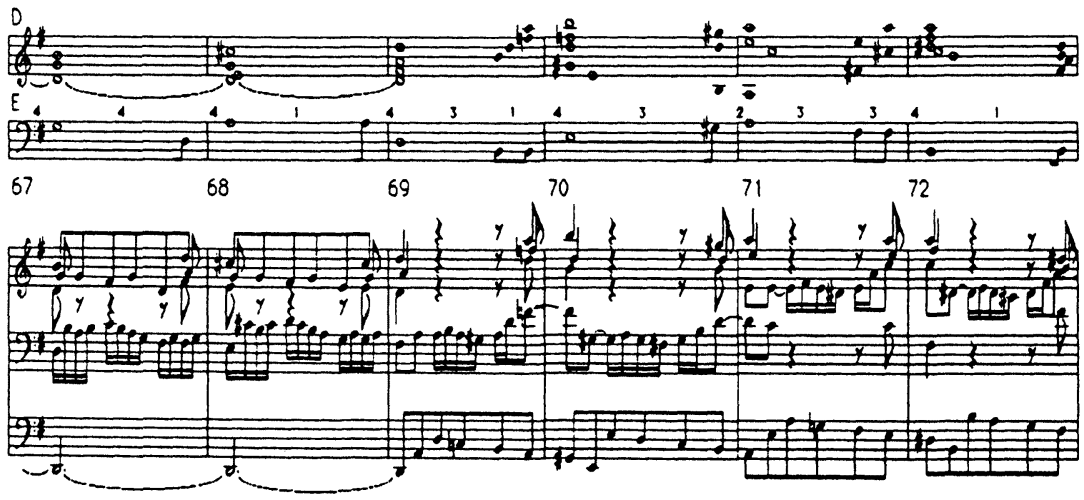


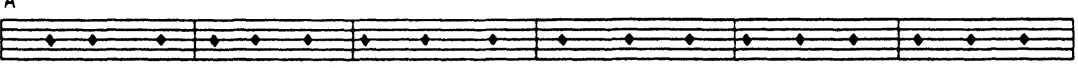

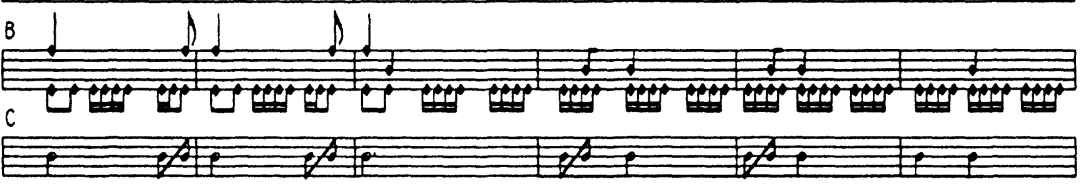
(a) (13)

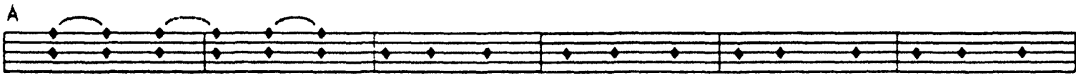

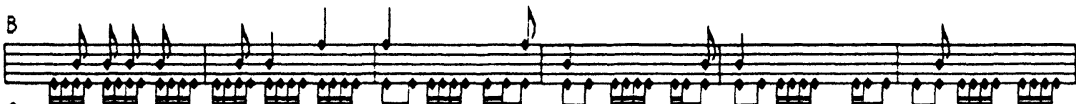
C

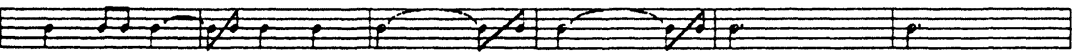
(a)
79
80
81
82
83
84

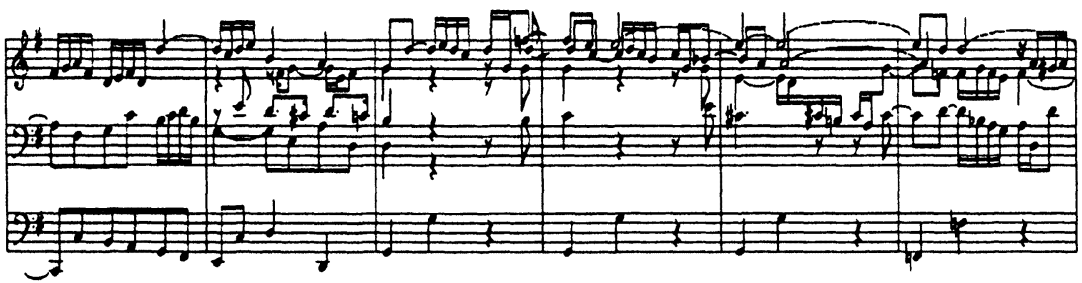


-VIII-
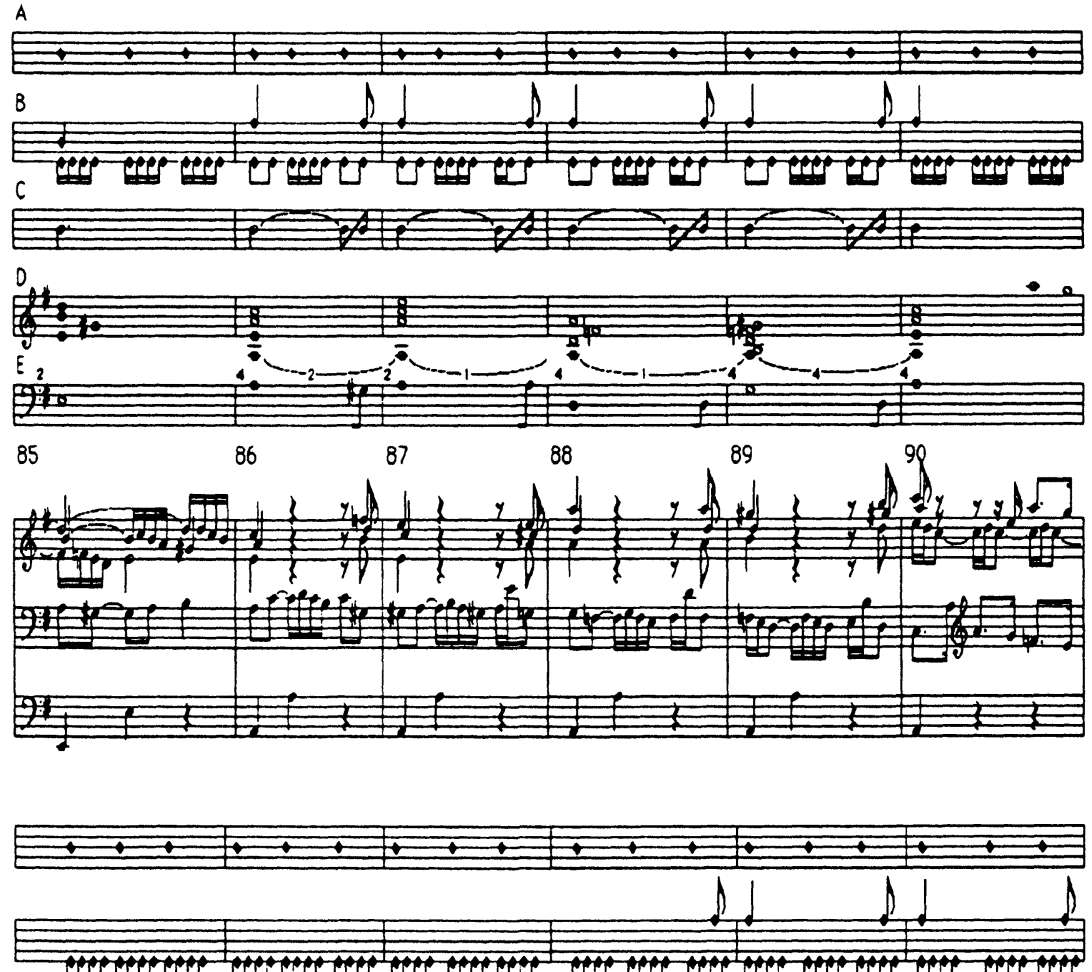

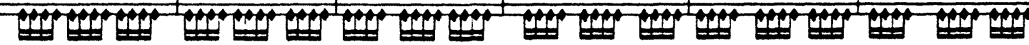
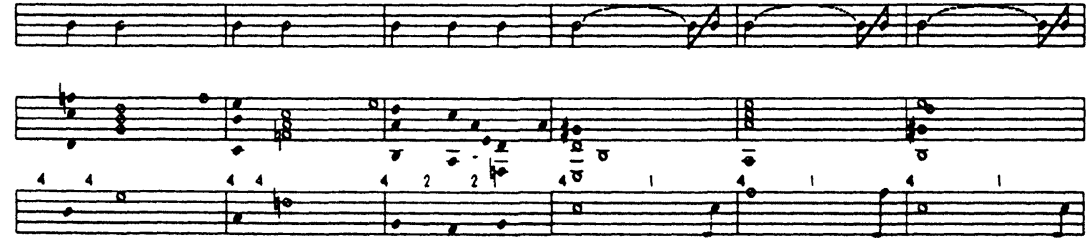
91
92
93
94
95
96

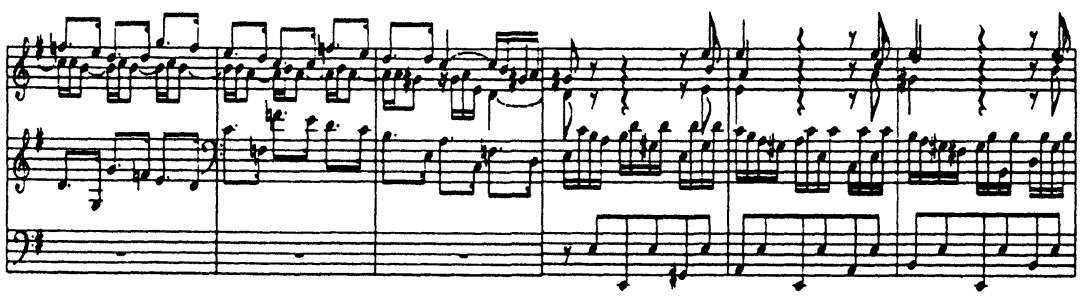


A
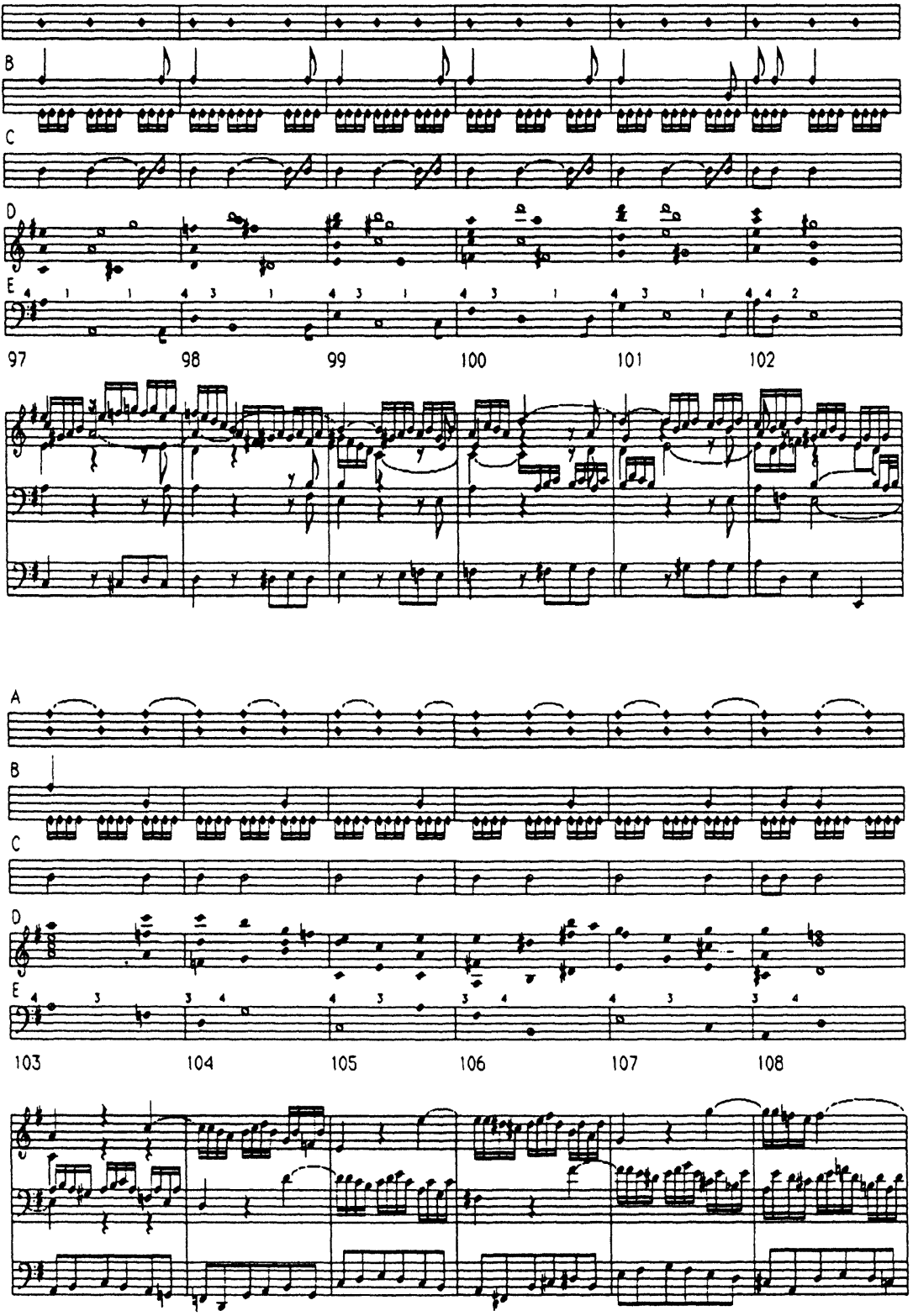
A

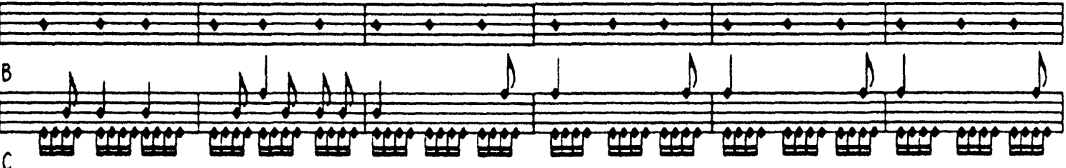

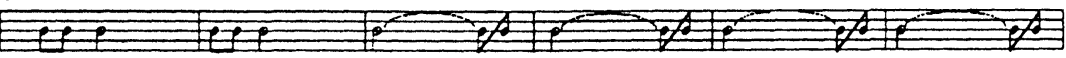

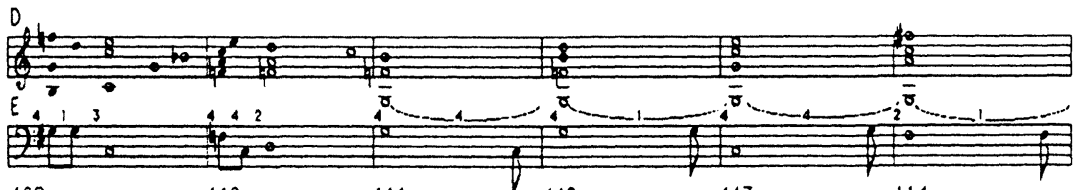

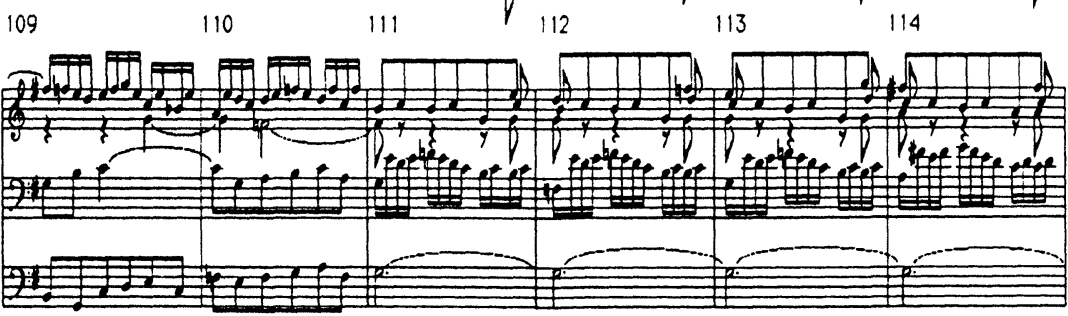

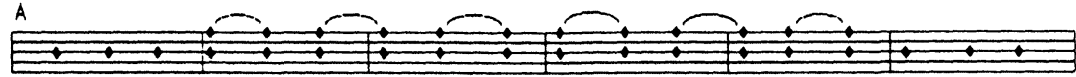
B

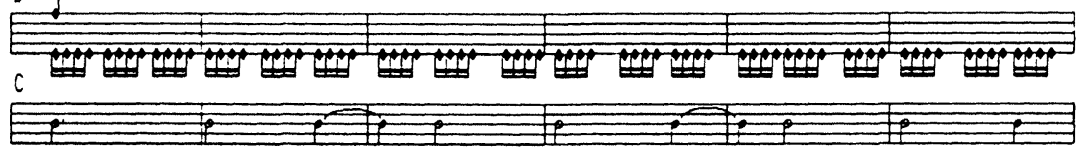
(2) 115

116

117

118

119

120

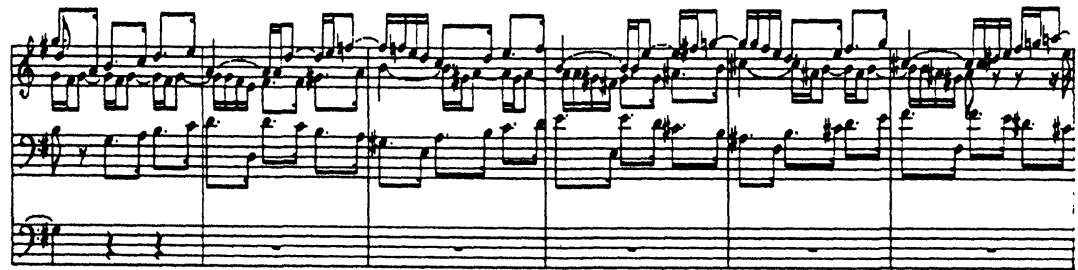


A
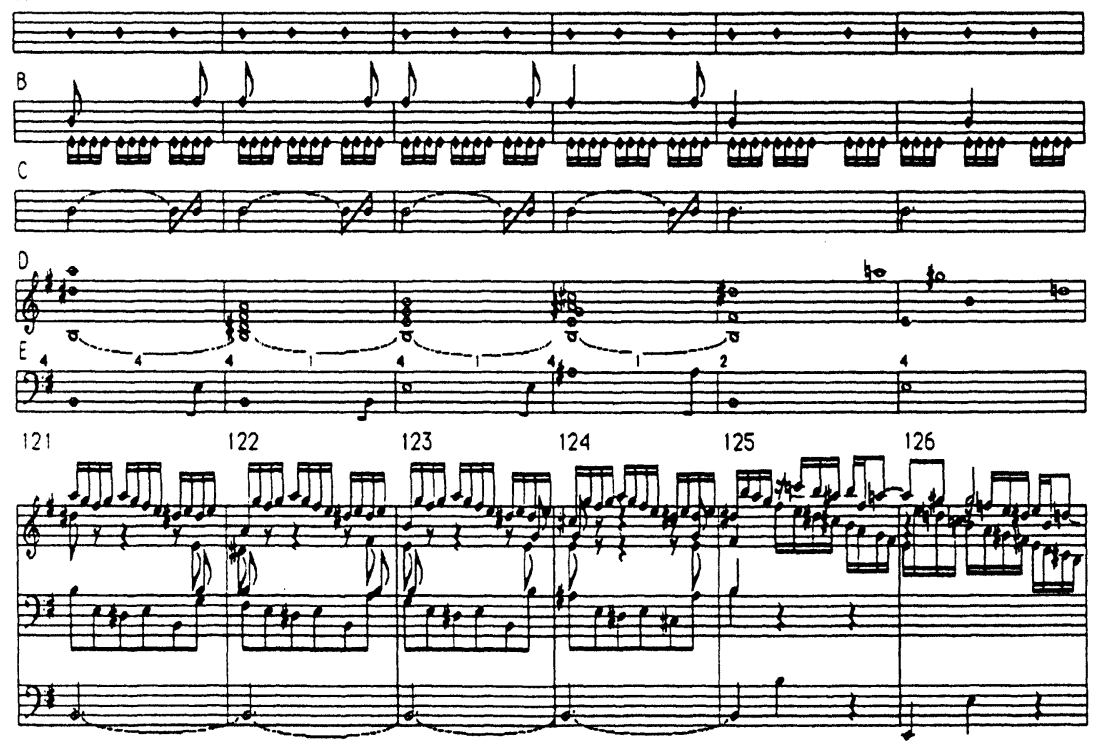

A

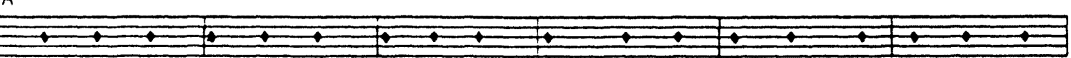
EC+10 C El$\mathrm{S}^{2}$ (27) 


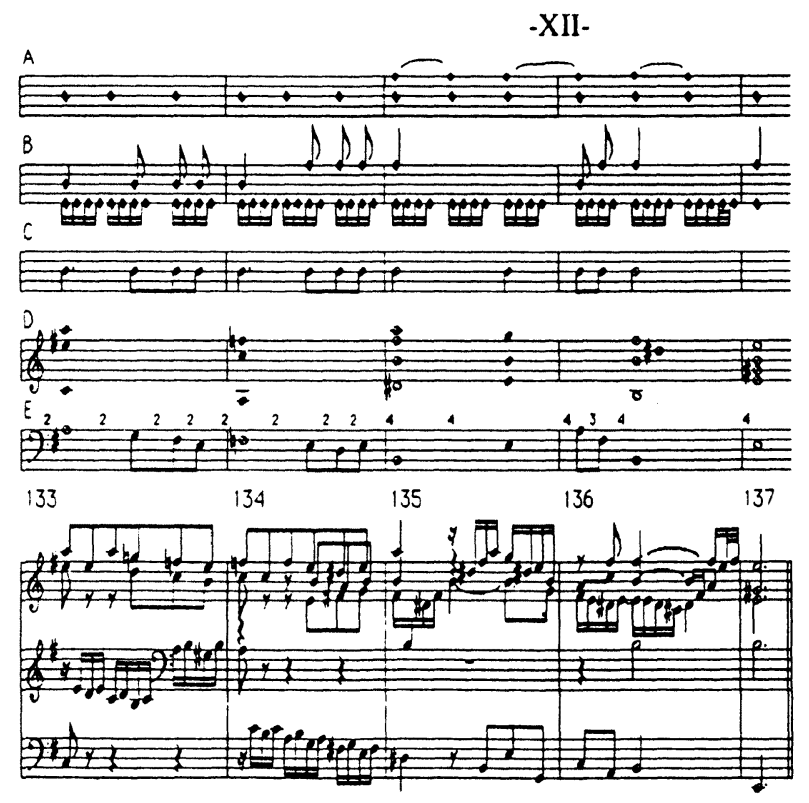

